\title{
CMOS voltage-controlled oscillator with high-performance MEMS tunable inductor
}

\author{
Uikyu Chae ${ }^{1,2}$, Jeongsoo Park ${ }^{3}$, Jeong-Geun Kim ${ }^{3}$, Hyun-Yong Y Y ${ }^{2}$ and Il-Joo Cho ${ }^{1,4,5^{*}}$
}

\begin{abstract}
LC CMOS voltage-controlled oscillators (VCOs) with tunable inductors are essential for high-performance, multi-band communication systems, such as loT applications and 5G communication. However, VCOs that use CMOS tunable inductors have difficulty in achieving high RF performance due to the low Q-factor of the inductor. In addition, previously reported CMOS VCOs integrated with MEMS inductors have used CMOS switches for tuning frequency bands, but they also had large signal losses on the switch. Herein, we propose a CMOS VCO that is integrated with a MEMS tunable inductor that tunes the frequency band with three MEMS switches. The proposed MEMS tunable inductor enables us to achieve high RF performance due to the suspended structure, and RF MEMS switches enable lower signal loss than CMOS switches. In this work, we successfully fabricated the proposed CMOS VCO integrated with a MEMS tunable inductor using the flip-chip bonding process, and we measured oscillation frequencies according to the actuation of the three switches. The oscillation powers were measured as -3.03 dBm @ $1.39 \mathrm{GHz}$, $-5.80 @$ $1.98 \mathrm{GHz},-7.44 \mathrm{dBm} @ 2.81 \mathrm{GHz}$, and - $8.77 \mathrm{dBm} @ 3.68 \mathrm{GHz}$.
\end{abstract}

Keywords: CMOS VCO, MEMS tunable inductor, RF MEMS

\section{Introduction}

Recently, communication technologies, such as wireless gigabit (wigig) [1] and the fifth-generation cellular network (5G) $[2,3]$ were developed to cover the increasing consumption of data. Also, various communication technologies, such as BLE, Zigbee, NFC, and WiFi, have been applied to multiple applications in the internet of things (IoT) application system [4]. These various communication technologies use different communicationfrequency bands, which makes it essential to develop a multi-band wireless transceiver that supports multiple frequency bands.

LC CMOS voltage-controlled oscillators (VCOs) with tunable inductors have been introduced to support multi-band communication systems [5-9]. However, CMOS-based inductors integrated on LC CMOS VCOs

\footnotetext{
*Correspondence: ijcho@kist.re.kr

${ }^{1}$ Center for BioMicrosystems, Brain Science Institute, Korea Institute of Science and Technology (KIST), 5 Hwarang-ro 14-gil, Seongbuk-gu, Seoul 02792, Republic of Korea

Full list of author information is available at the end of the article
}

had a relatively high signal loss due to the low thickness of the metal and the loss of the substrate. Therefore, MEMS-based inductors were introduced to reduce signal loss by increasing the thickness of metal in the inductor and by increasing the gap between the inductor and the substrate [10-12]. However, the previously reported VCOs integrated with MEMS inductors used CMOSbased RF switches to tune inductances have higher insertion loss and lower isolation than RF MEMS switches, which meant that the VCOs could not be used for high performance RF systems [13, 14].

In this paper, we propose a CMOS VCO integrated with a MEMS tunable inductor for tuning multiple frequency bands with low signal loss. To achieve a wide tuning range, we designed the MEMS tunable inductor as a discrete type [15-18], which varies the length of the inductor using RF MEMS switches. We designed the proposed VCO to achieve a wide tuning range of four frequency bands through integration of the MEMS tunable inductor. The frequency of the tunable inductor is tuned by three electrostatically actuated RF MEMS switches 
and also fine-tuned by the varactor in CMOS VCO. In this manuscript, we successfully integrated the fabricated tunable inductor on a CMOS VCO using the flipchip bonding process, and we demonstrated the tuning of four oscillation frequencies by actuating the RF MEMS switches of the MEMS tunable inductor.

\section{Operation principle and design}

Figure 1 shows the principle of operation of the proposed VCO with an integrated MEMS tunable inductor. Our proposed CMOS VCO was a complementary crosscoupled type of oscillator, and it generated the oscillation frequency by changing the values of the inductance and capacitance [9] (Fig. 1a). The three electrostatically actuated RF MEMS switches (i.e., SW 1-3) integrated in the MEMS tunable inductor are actuated to change the effective length of the inductor into four different values by pulling the membrane down by electrostatic forces when the actuation voltage is applied to the bottom electrode under the membrane (Fig. 1b). When the SW3 is ON, the effective length of the inductor goes to minimum values while the effective length changes to the maximum value when all of the switches are in the OFF states. (Fig. 1c). Thus, the oscillation frequency generated by the VCO can be tuned extensively according to the switching states of the MEMS tunable inductor (Fig. 1d).

The proposed VCO consists of a CMOS VCO and the MEMS tunable inductor, and the suspended inductor is a 3D-shaped inductor with three RF MEMS switches for changing the effective length of the inductor (Fig. 2a, b). The MEMS inductor was designed as a differential structure to obtain a high $Q$ value with a small size [19]. The structure of the MEMS inductor was suspended from the substrate and connected to a bottom metal line only through anchors to minimize the parasitic capacitance between the substrate and the suspended inductor. The RF MEMS switch integrated on the tunable inductor consists of a suspended membrane, a dielectric layer, and the bottom electrodes. An actuation voltage is applied between the membrane and the bottom layer to move the membrane downward. The Au contact metal under the dielectric layer connects the separated bottom electrodes of the MEMS inductor (Fig. 2c). The MEMS tunable inductor was designed to be integrated with a CMOS VCO by electrically connecting the metal line MEMS inductor to the signal line of the CMOS VCO through $\mathrm{Au}$ bumps (Fig. 2d).

\section{Fabrication process}

Figure 3 shows the process of fabricating the proposed tunable inductor integrated with the VCO. The starting wafer was a silicon wafer on which we grew a thermal oxide layer to reduce the loss of RF signals to the substrate. First, $0.5 \mu \mathrm{m}$ of $\mathrm{Ti} / \mathrm{Au}$ was deposited for both the bottom electrodes of the switches and the bottom metal line of an inductor using a lift-off process (Fig. 3a). Then, a $3-\mu \mathrm{m}$-thick polyimide (PI) was spun and cured as a sacrificial layer to make the suspended cantilever-type switch and inductor structures. The thickness of the PI determines the air gap of the MEMS tunable inductor as well as the initial gap between the bottom metal and the contact metal of the switch. Accurate control of the air

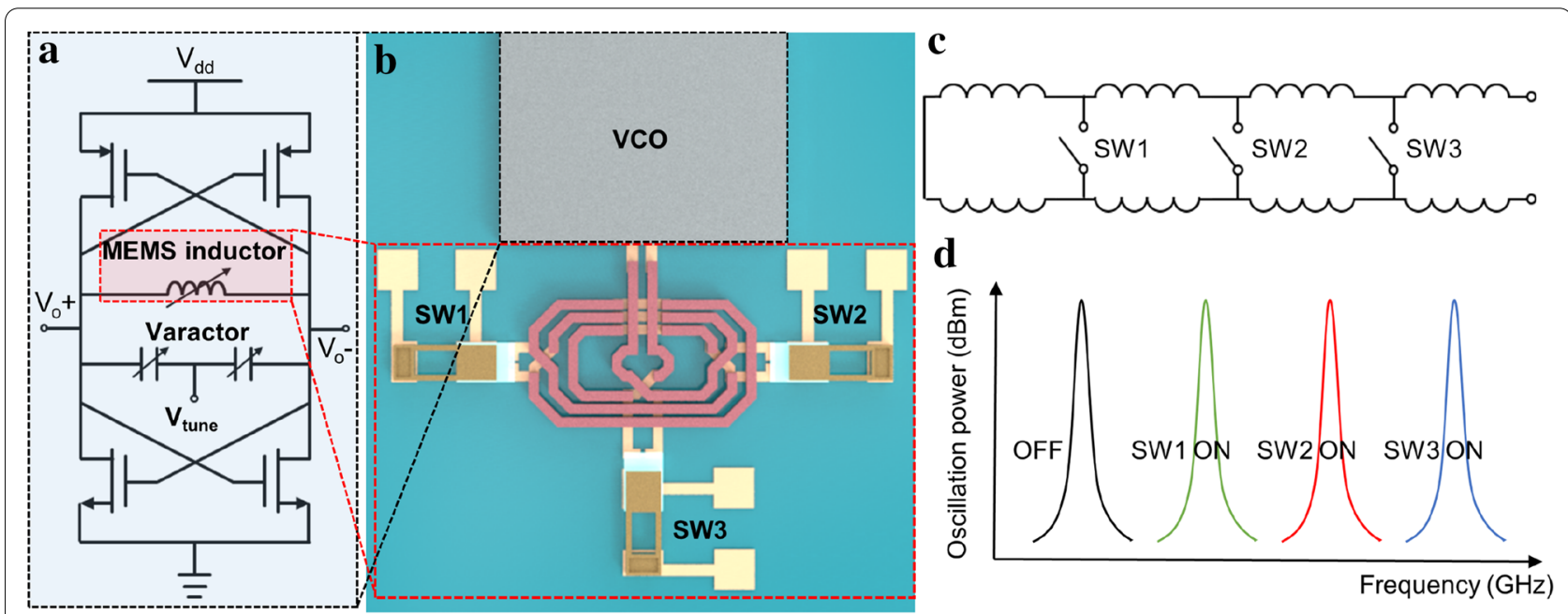

Fig. 1 Schematic illustration of the operation of the MEMS tunable inductor. a Circuit sche-matic of a CMOS VCO integrated with the MEMS tunable inductor. $\mathbf{b}$ Conceptual diagram of a VCO integrated with the MEMS tunable inductor operating at four frequency bands. The effective length of the inductor changes with the switching state. $\mathbf{c}$ The equivalent circuit model of the MEMS tunable inductor. $\mathbf{d}$ Power spectrum according to the switching states of the MEMS tunable inductor 

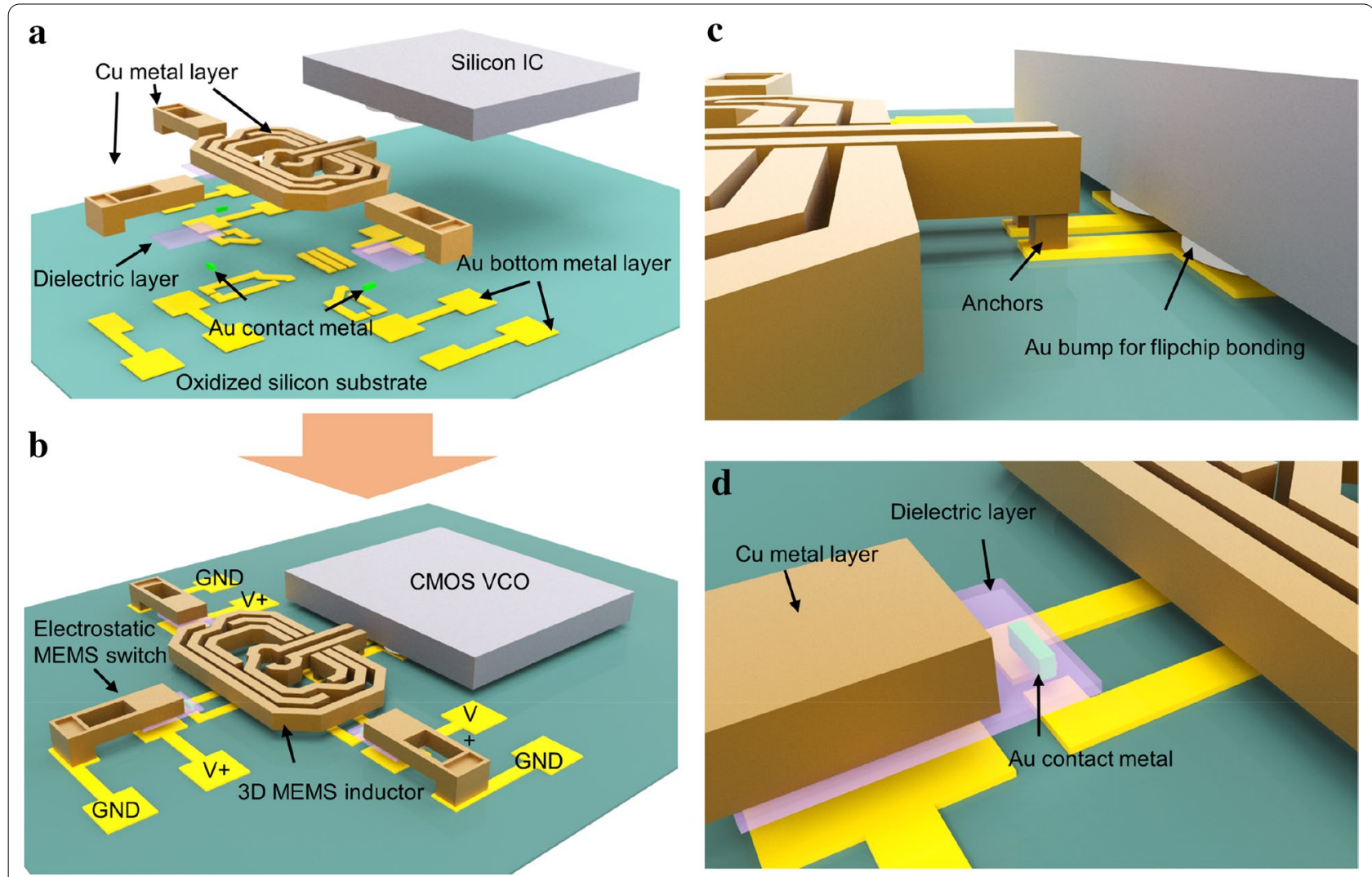

Fig. 2 Schematic diagrams of the proposed VCO integrated with the MEMS tunable inductor. a Schematic diagram of each component layer of the proposed VCO. $\mathbf{b}$ Schematic diagram of the proposed VCO composed of the MEMS tunable inductor with three electrostatically actuated RF MEMS switches, a 3D MEMS inductor, and a CMOS VCO. c Close-in image of the RF MEMS switch with a contact metal and bottom metal layer of the MEMS inductor. $\mathbf{d}$ Close-up image of the anchors that connected the MEMS inductor and the CMOS VCO

gap is very important because it is related to the signal isolation value during the OFF state of the switch as well as the Q-factor of the inductor. The cured PI was patterned for forming dimples of contact metal that ensure reliable contact between the bottom metal and the contact metal. Next, we deposited a $0.5 \mu$ m layer of $\mathrm{Au} / \mathrm{Cr}$ for the contact metal using a lift-off process (Fig. 3b), and this was followed by the deposition of $0.2 \mu \mathrm{m}$ of the SiN dielectric layer using plasma enhanced chemical vapor deposition (PECVD). After patterning the dielectric layer, we patterned the PI to form the anchors of the switches and an inductor (Fig. 3c). Then, we deposited a $0.2 \mu \mathrm{m}$ layer of $\mathrm{Ti} / \mathrm{Cu}$ as a seed layer for electroplating the thick metal layer, and we electroplated $4 \mu \mathrm{m}$ of $\mathrm{Cu}$ as both the membrane of switches and an inductor using a photoresist as a mold (Fig. 3d). The thick metal layer helps to enhance the mechanical stability of the switch and reduce the electrical resistance of the inductor for achieving a high Q-factor. Then, the seed layer was removed using wet etchants, and the sacrificial layer was removed using an $\mathrm{O}_{2}$ plasma asher. Next, the tunable inductor and $\mathrm{RF}$ switches were released successfully as shown in Fig. 3e.
A CMOS VCO was fabricated independently using a commercial 1-poly 6-metal, $0.18-\mu \mathrm{m}$ CMOS technology [9]. Finally, we integrated the variable inductor with the CMOS VCO using a flip-chip bonding process. For the bonding process, we deposited Au stud bumps on the VCO pads and connected the pads of the VCO pads to the pads of the tunable inductor through the flip-chip bonding process (Fig. 3f).

As shown in Fig. 4, we successfully fabricated the CMOS VCO integrated with the MEMS tunable inductor. SEM images of the VCO that was fabricated shows that three cantilever-type RF MEMS switches, the CMOS VCO, and the suspended inductor were integrated successfully in a single die. The size of the tunable inductor was $1200 \times 600 \mu \mathrm{m}$, and it consisted of three RF MEMS switches of $170 \times 80 \mu \mathrm{m}$. In the MEMS inductor that was fabricated, the suspended structures were connected to the bottom metals with anchors (Fig. 4a). Also, the contact metal of the switch that was fabricated was formed beneath the dielectric layer that was used to prevent an electrical connection between the bottom metal and the membrane when the switch is in the ON state. The initial 

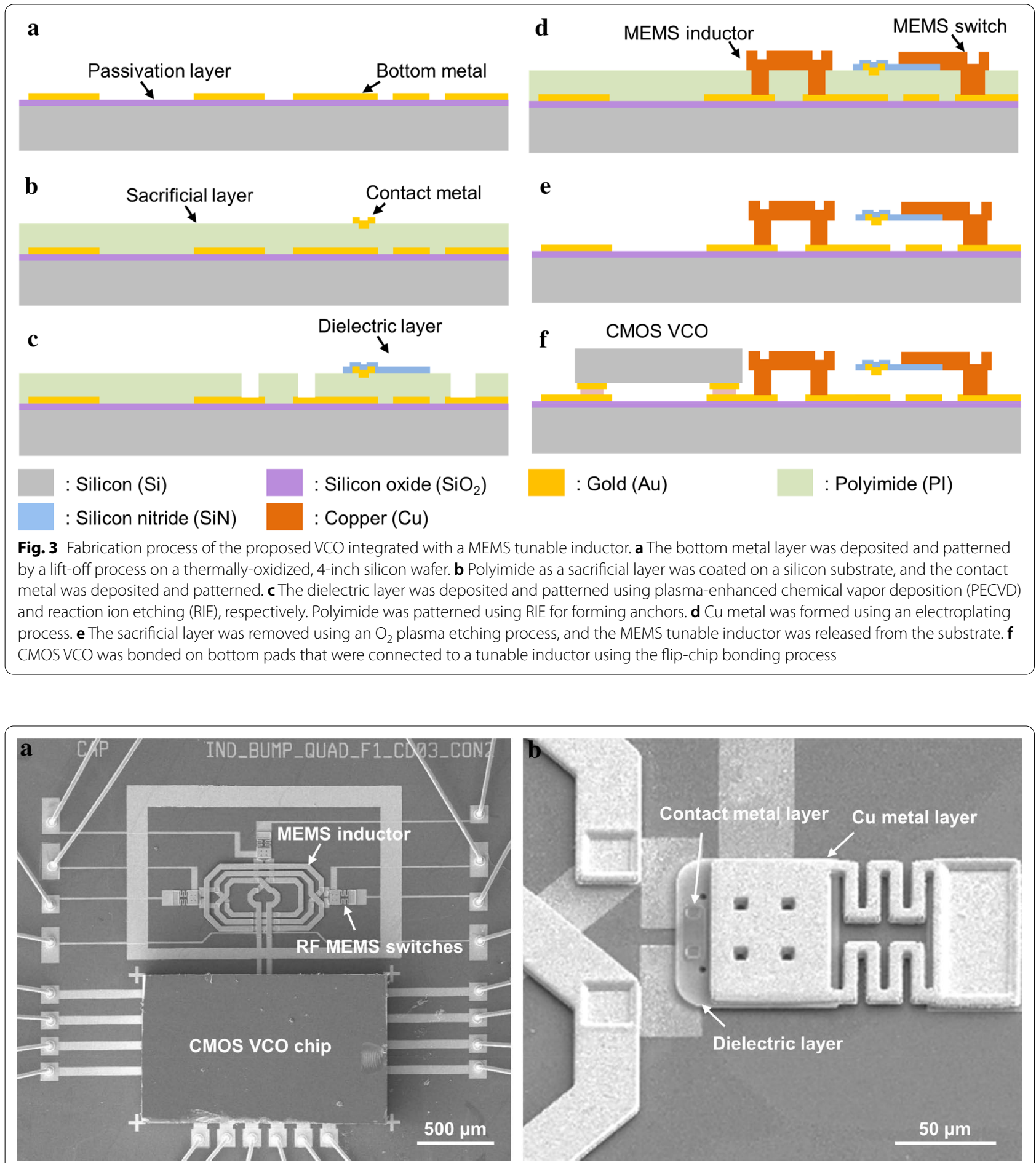

Fig. 4 Scanning electron microscopy (SEM) images of the fabricated CMOS VCO integrated with the MEMS tunable inductor. a A SEM image of the fabricated inductor with CMOS VCO chip and pads. b A close-in image of the electrostatic RF MEMS switch and signal lines connected to MEMS inductor

gap between the contact metal and the metal of the bottom metal was about $3 \mu \mathrm{m}$, which was determined by the thickness of the PI. The switch that was fabricated had a meander structure with beams that were $6 \mu \mathrm{m}$ wide, which enabled it to reduce the actuation voltage below $20 \mathrm{~V}$ (Fig. 4b). 


\section{RF characteristics}

First, we measured the insertion loss and isolation of the MEMS switch in the fabricated MEMS tunable inductor. When the switch was $\mathrm{ON}$ state, the insertion losses were $-0.221 \mathrm{~dB}$ at $2.4 \mathrm{GHz}$ and $-0.150 \mathrm{~dB}$ at $5 \mathrm{GHz}$, and when the switch was OFF state, the isolation were measured as $-42.89 \mathrm{~dB}$ at $2.4 \mathrm{GHz}$ and $-43.19 \mathrm{~dB}$ at $5 \mathrm{GHz}$ (Fig. 5a). In addition, we evaluated the long term reliability of the switch, and we confirmed that the switch maintained the performance for more than 100 million cycles (Fig. 5b). We also measured the Q-factor and inductance of the MEMS inductor. When all switches were in the OFF state, the Q-factors was measured as 15.6 at $2.4 \mathrm{GHz}$ and 20.77 at $5 \mathrm{GHz}$, and the inductance was measured as $5.27 \mathrm{nH}$ at $2.4 \mathrm{GHz}$ and 6.08 at $5 \mathrm{GHz}$ (Fig. 6).

Finally, we measured the oscillation frequency and oscillation power by operating the fabricated CMOS VCO integrated with the MEMS tunable inductor. When all switches of the MEMS tunable inductor were in the OFF state, the oscillation frequency of the VCO was $1.39 \mathrm{GHz}$, and the oscillation power was $-3.03 \mathrm{dBm}$. Then, we sequentially changed the state of switches 1 , 2 , and 3 to the ON state and measured that the oscillation power and frequency were $-5.80 @ 1.98 \mathrm{GHz}$, - 7.44 dBm @ $2.81 \mathrm{GHz}$, and - $8.77 \mathrm{dBm} @ 3.68 \mathrm{GHz}$, respectively (Fig. 7). We confirmed that the oscillation power decreased as the oscillation frequency increased by changing the state of switches. These results successfully showed that the fabricated VCO integrated with the MEMS tunable inductor could be tuned to four different frequencies, and the VCO can be applied to mobile communication devices and RF circuits that require multiband oscillation frequencies.

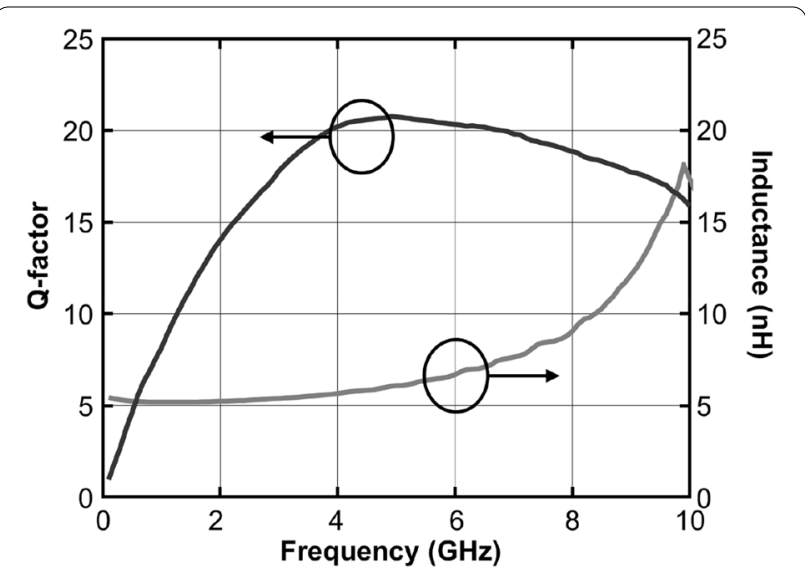

Fig.6 Measured inductance and Q-factor of fabricated MEMS tunable inductor at various signal frequencies

\section{Conclusions}

In this paper, we proposed and fabricated a VCO integrated with a MEMS tunable inductor that tuned a wide range of frequency bands by electrostatically actuating three RF MEMS switches to achieve high RF performances. We fabricated the MEMS tunable inductor successfully and integrated it with a CMOS VCO through the flip-chip bonding process. We could implement a discrete-type tunable inductor by utilizing three RF MEMS switches to vary the effective length of the inductor. Thus, the proposed VCO operated to tune a wide range according to the switching state of RF MEMS switches and the applied voltages. We also designed a meander-type bridge of the RF MEMS switch to achieve a low actuation voltage, i.e., less than $20 \mathrm{~V}$.
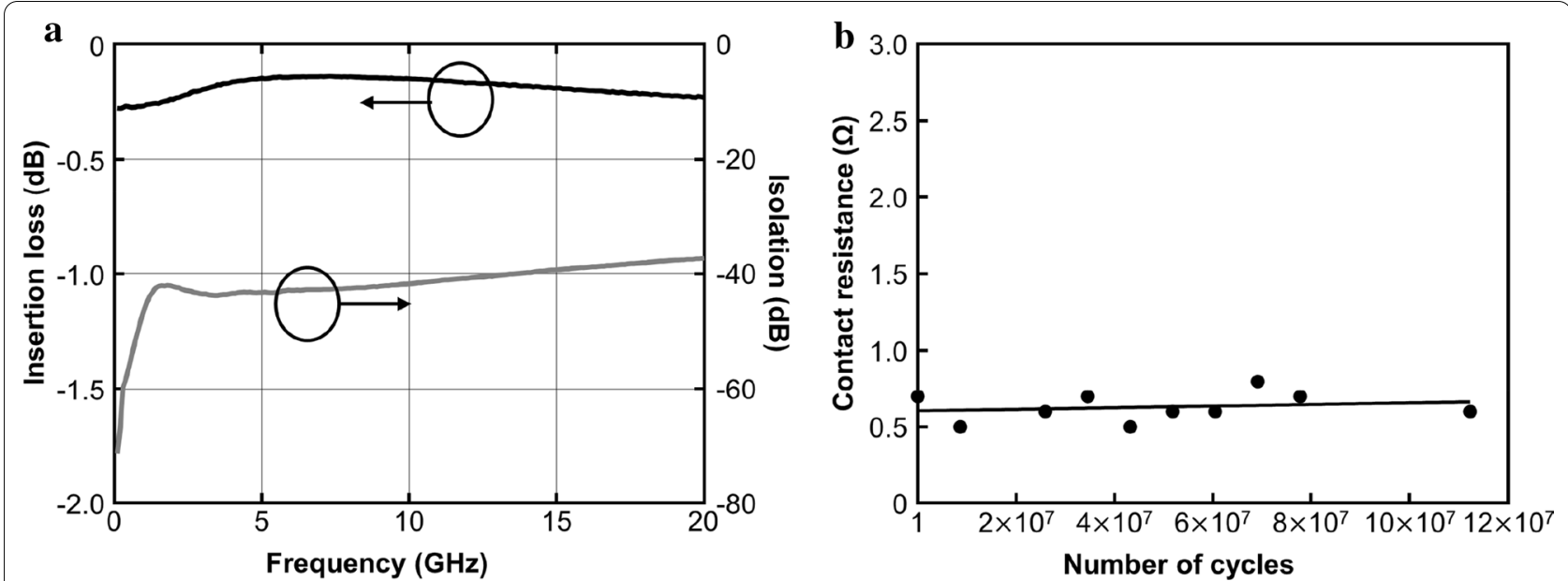

Fig. 5 Measured RF characteristics of the RF MEMS switch integrated in the fabricated MEMS tunable inductor. a Insertion loss and Isolation values of the switch at various signal frequencies. $\mathbf{b}$ Contact resistance according to the number of switching times 

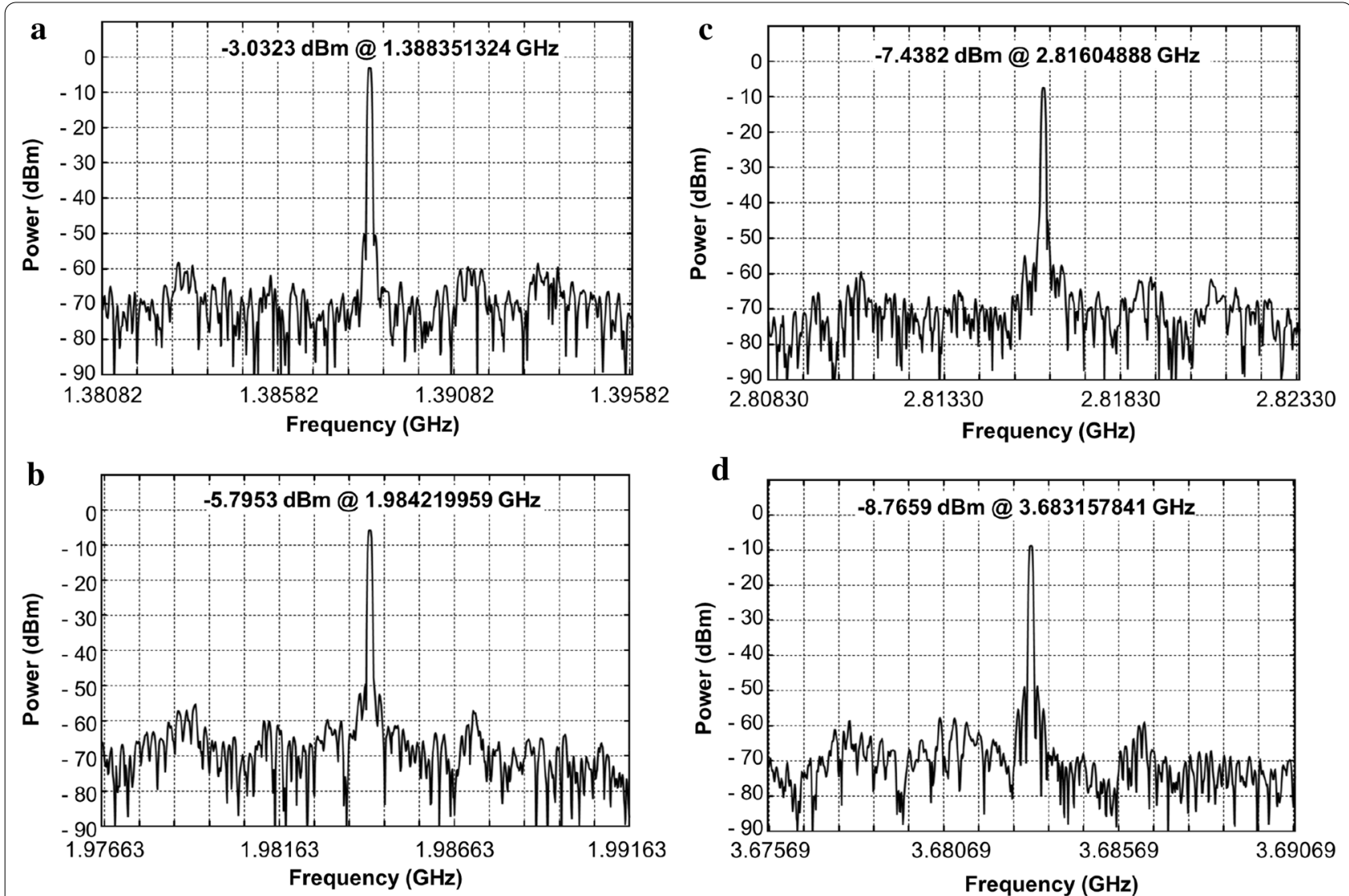

Fig. 7 Power spectra which show the oscillation frequency according to the operation of the tunable inductor. a When all switches were OFF, the oscillation frequency was $1.39 \mathrm{GHz}$ and the power was $-3.03 \mathrm{dBm}$. b When switch 1 was ON, the oscillation frequency was $1.98 \mathrm{GHz}$, and the power was - $5.80 \mathrm{dBm}$. c When switch 2 was in the ON state, the oscillation frequency was $2.82 \mathrm{GHz}$, and the power was - 7.44 $\mathrm{dBm}$. $\mathbf{d}$ When the switch 3 was in the ON state, the oscillation frequency was $3.68 \mathrm{GHz}$, and the power was $-8.77 \mathrm{dBm}$

By operating the CMOS VCO integrated with the MEMS tunable inductor, we successfully measured the oscillation frequency and oscillation power, which demonstrated the potential applicability of the CMOS VCO to multi-band communication systems that require high RF performances and a wide tuning range.

\section{Abbreviations \\ CMOS: Complementary Metal-Oxide-Semiconductor; MEMS: Microelectro- mechanical Systems; VCO: Voltage-controlled oscillator; loT: Internet of things; wigig: Wireless gigabit; 5G: Fifth-generation cellular network; BLE: Bluetooth; NFC: Near field communication; WiFi: Wireless fidelity; RF: Radio frequency.}

\section{Acknowledgements}

This work was supported by the Brain Research Program of the National Research Foundation (NRF) funded by the Korean government (MSIT) (NRF2017M3C7A1028854). Also, this work was supported by the Brain Convergence Research Program of the National Research Foundation (NRF) funded by the Korean government (MSIT) (NRF-2019M3E5D2A01063814), Bio \& Medical Technology Development Program of the National Research Foundation (NRF) funded by the Korean government (MSIT) (NRF-2017M3A9B3061319), the Institute for Basic Science (IBS-R001-D2), and Korea Institute of Science and Technology (KIST) intramural Grant (2E30963, MI).

\section{Authors' contributions}

UC performed most of the experiments, fabricated the MEMS device, prepared the figures, and wrote the manuscript. JP and J-GK were involved in designing and fabricating CMOS devices and performed the experiments. $\mathrm{H}-\mathrm{YY}$ discussed the results. I-JC reviewed and edited the manuscript. All authors read and approved the final manuscript.

\section{Funding}

The Brain Research Program of the National Research Foundation (NRF) funded by the Korean government (MSIT) (NRF-2017M3C7A1028854); The Brain Convergence Research Program of the National Research Foundation (NRF) funded by the Korean government (MSIT) (NRF2019M3E5D2A01063814); Bio \& Medical Technology Development Program of the National Research Foundation (NRF) funded by the Korean government (MSIT) (NRF-2017M3A9B3061319); the Institute for Basic Science (IBS-R001-D2); Korea Institute of Science and Technology (KIST) intramural Grant (2E30963, $\mathrm{MI})$.

Availability of data and materials

All data produced and analyzed in this study are included in this article.

\section{Declarations}

\section{Competing interests}

The authors declare that they have no competing interests. 


\section{Author details}

${ }^{1}$ Center for BioMicrosystems, Brain Science Institute, Korea Institute of Science and Technology (KIST), 5 Hwarang-ro 14-gil, Seongbuk-gu, Seoul 02792, Republic of Korea. ${ }^{2}$ School of Electrical Engineering, Korea University, 145 Anam-ro, Seongbuk-gu, Seoul 02841, Republic of Korea. ${ }^{3}$ Department of Electronic Engineering, Kwangwoon University, 20 Gwangun-ro, Nowon-gu, Seoul 01897, Republic of Korea. ${ }^{4}$ School of Electrical and Electronics Engineering, Yonsei University, 50 Yonsei-ro, Seodaemun-gu, Seoul 03722, Republic of Korea. ${ }^{5}$ Yonsei-KIST Convergence Research Institute, Yonsei University, 50 Yonsei-ro, Seodaemun-gu, Seoul 03722, Republic of Korea.

Received: 14 July 2021 Accepted: 17 November 2021

Published online: 26 November 2021

\section{References}

1. Hansen CJ (2011) WiGiG: multi-gigabit wireless communications in the $60 \mathrm{GHz}$ band. IEEE Wireless Commun 18:6-7

2. Xiang W, Zheng K, Shen XS (2016) 5 G mobile communications. Springer, Cham

3. Osseiran A, Monserrat JF, Marsch P (2016) 5G mobile and wireless communications technology. Cambridge University Press

4. Gubbi J, Buyya R, Marusic S, Palaniswami M (2013) Internet of things (loT): a vision, architectural elements, and future directions. Fut Gener Comput Syst 29:1645-1660

5. Xian L, Wenyuan L, Zhigong W (2010) A wide tuning range LC-VCO using switched capacitor array technique. In: 2010 international symposium on signals, systems and electronics. IEEE, Nanjing

6. Sánchez-Azqueta C, Aguirre J, Gimeno C, Aldea C, Celma S (2015) A 1.7-GHz wide-band CMOS LC-VCO with 7-Bit coarse control. In: 2015 IEEE international symposium on circuits and systems (ISCAS). IEEE, Lisbon

7. Li Z, Kenneth OK (2004) A 1-V low phase noise multi-band CMOS voltage controlled oscillator with switched inductors and capacitors. In: 2004 IEEE radio frequency integrated circuits (RFIC) systems. Digest of Papers. IEEE, Forth Worth, TX

8. Min B, Jeong H (2005) 5-GHz CMOS LC VCOs with wide tuning ranges. IEEE Microw Wireless Comp Lett 15:336-338

9. Kim J, Park J, Kim J-G (2018) A low phase noise multi-band LC VCO using a switched differential inductor. IEICE Elect Express 15:1-7

10. Park E-C, Choi Y-S, Yoon J-B, Hong S (2003) Fully integrated low phasenoise VCOs with on-chip MEMS inductors. IEEE Trans Microw Theory Tech 51:289-296

11. Tseng S-H, Hung Y-J, Juang Y-Z, Lu MS-C (2007) A 5.8-GHz VCO with CMOS-compatible MEMS inductors. Sens Actuators A Phys 139:187-193

12. Yoon J-B, Kim B-I, Choi Y-S, Yoon E (2002) 3-D lithography and metal surface micromachining for RF and microwave MEMS. In: Technical Digest. MEMS 2002 IEEE International Conference. In: Fifteenth IEEE international conference on micro electro mechanical systems (Cat. No.02CH37266). IEEE, Las Vegas, NV

13. Rebeiz GM, Muldavin JB (2001) RF MEMS switches and switch circuits. IEEE Microw Mag 2:59-71

14. Rebeiz GM, Patel CD, Han SK, Ko C-H, Ho KMJ (2013) The search for a reliable MEMS switch. IEEE Microw Mag 14:57-67

15. Zhou S, Sun X-Q, Carr WN (1999) A monolithic variable inductor network using microrelays with combined thermal and electrostatic actuation. J Micromech Microengineering 9:45-50

16. Park P, Kim CS, Park MY, Kim SD, Yu HK (2004) Variable inductance multilayer inductor with MOSFET switch control. IEEE Electron Device Lett 25:144-146

17. Lee S, Kim JM, Kim JM, Kim YK, Kwon Y (2005) Millimeter-wave MEMS tunable low pass filter with reconfigurable series inductors and capacitive shunt switches. IEEE Microw Wireless Compt Lett 15:691-693

18. Rais-Zadeh M, Kohl PA, Ayazi F (2008) MEMS switched tunable inductors. J Microelectromech Syst 17:78-84

19. Reiha MT, Choi T-Y, Jeon J-K, Mohammadi S, Katehi LPB (2003) High-Q differential inductors for RFIC design. In: 2003 33rd European Microwave Conference. IEEE, Munich

\section{Publisher's Note}

Springer Nature remains neutral with regard to jurisdictional claims in published maps and institutional affiliations.

\section{Submit your manuscript to a SpringerOpen ${ }^{\circ}$ journal and benefit from:}

- Convenient online submission

- Rigorous peer review

- Open access: articles freely available online

- High visibility within the field

- Retaining the copyright to your article

Submit your next manuscript at $\boldsymbol{\nabla}$ springeropen.com 